\title{
Worriers versus Warriors: Tailoring mHealth to Address Differences in Patients with Chronic Arthritis
}

\author{
Jonas Geuens \\ Department of Computer Science \\ KU Leuven \\ Leuven, Belgium \\ jonasgeuens@gmail.com
}

\author{
Rene Westhovens \\ Faculty of Medicine \\ $K U$ Leuven \\ City, Country \\ rene.westhovens@uzleuven.be
}

\author{
Thijs Swinnen \\ Department of Rehabilitation Sciences \\ KU Leuven \\ Leuven, Belgium \\ thijs.swinnen@uzleuven.be
}

\author{
Robin De Croon \\ Department of Computer Science \\ KU Leuven \\ Leuven, Belgium \\ robin.decroon@kuleuven.be
}

\author{
Luc Geurts \\ Department of Electrical Engineering \\ $K U$ Leuven \\ Leuven, Belgium \\ luc.geurts@kuleuven.be
}

\author{
Vero Vanden Abeele \\ Department of Computer Science \\ KU Leuven \\ Leuven, Belgium \\ vero.vandenabeele@kuleuven.be
}

\begin{abstract}
Chronic arthritis (CA) represents a group of musculoskeletal diseases that require frequent physical therapy and a well-monitored medication regime to improve or maintain disease status. To support patients in this disease management, health apps may provide a solution. However, patients with $\mathrm{CA}$ differ in their disease trajectory, needs and preferences. Therefore, in this paper, we investigate the tailoring of motivational mHealth features for the heterogeneous group of patients with CA. An exploratory study was carried out, consisting of semistructured interviews and focus groups with 34 patients with CA and six health professionals. Through qualitative analysis, we first derive a typology distinguishing patients of the Defeatist, Worrier, Warrior and Cruiser type. Each type is characterized by their own disease management needs and coping strategies. Next, we unveil how each patient type has different preferred motivational strategies. These findings may inform healthcare informatics researchers who wish to tailor mHealth applications and interventions to the diversity of patients characterized by musculoskeletal diseases and chronic pain.
\end{abstract}

Index Terms-Chronic arthritis, mHealth, personalization, tailoring, motivational design, patient profiles

\section{INTRODUCTION}

Chronic Arthritis (CA) is an umbrella term for inflammatory diseases that affects $22.7 \%$ of the US and $23.7 \%$ of the EU population [1]. Symptoms include joint pain, swelling, stiffness and joint destruction, resulting in progressive immobility [2] and chronic pain. Effective drugs to treat CA are available but require a long-term commitment. In addition, patients are recommended to participate in physical activity to improve mobility, cardiovascular endurance, movement control and muscle strength. Moreover, patients are required to keep a close watch on their disease parameters in order to identify changes and adapt medication intake or exercise regimen timely. In sum, managing $\mathrm{CA}$ is a lifelong, complex and demanding activity. Understandably, patients often fail to comply with this enduring treatment regimen [3]-[5].

A possible (partial) solution to support patients with CA in their disease management may come in the shape of health apps that include features to support and motivate them to perform behaviors that promote disease management. The use of mHealth and self-care applications has seen a worldwide increase [6]. Specifically for arthritis patients, myriad applications exist [7]-[9] to support them in disease management. However, research on long-term adoption of these apps for patients with arthritis is lacking. In general, research suggests that the adoption of self-care technology is suboptimal [10], and that compliance to eHealth interventions is low [11]. One limiting factor may be the generic nature of many of these selfcare apps and their inability to tailor features at the individual level. In the domain of mHealth, it has been convincingly argued that a one-size-fits-all approach is not sufficient, and that tailoring motivational features or persuasive strategies to user types [12]-[14] or patient group [15] is warranted. Thus far, most tailoring of such features has centered on personality traits or gamer types or existing health behavior models. To the best of the authors' knowledge, the tailoring of motivational features towards specific patient typologies, characterized by chronic pain and musculoskeletal disease, has not yet received attention. Therefore, this paper reports on an exploratory investigation of patients with $\mathrm{CA}$ and health professionals, and their preferences for motivational features.

Our first contribution is theoretical; through a qualitative analysis of semi-structured interviews and focus groups with patients and health professionals, we introduce four different CA patient types (Defeatist, Worrier, Warrior and Cruiser) and establish their relevance in the context of healthcare informatics. Our second contribution is more applied, we demonstrate 
how each patient type aligns with different preferences for motivational strategies, and how this can inform the design of personalized future interactive applications.

\section{BACKGROUND}

In this section, we present more information on CA and the different methods used by health professionals to typify patients with CA, on the basis of disease activity, pain and activity avoidance versus persistence. Next, we present current persuasive strategies, and how these have been tailored towards different users. Finally, we end with related work by other researchers in the domain of mHealth, self-care and CA.

\section{A. Profiling Patients with Chronic Arthritis}

CA is characterized by its fluctuating and progressive nature; inflammations come and go but gradually worsen over time. Therefore, every person with CA has a unique trajectory; some achieve stabilization of disease progression early on, others continue deteriorating at a rapid pace [16]. Besides a loss in mobility, pain is also characteristic of the disease. Acute musculoskeletal pain is triggered by an inflammation of joint or vertebra. But the pain often persists after the acute phase has waned. This chronic pain, defined as pain that persists longer than three months [17], may trigger secondary effects such as fatigue, anxiety, and even depression.

Given the diversity of CA disease trajectories, providing the same treatment to every person is neither effective nor feasible, and may even impede recovery [18]. To tailor interventions, health professionals have investigated how to profile patients with $C A$. Below we discuss different models used in CA patient profiling, along with the associated instruments.

1) Profiling on the basis of disease activity: To better understand disease activity and to control for phases of inflammation, patients with $\mathrm{CA}$ are regularly requested to provide a Disease Activity Score (DAS), where they are asked to indicate the number of tender or swollen joints (typically out of 28). In combination with a global health assessment and blood markers of inflammation [19], values are combined into one score between 0 and 10. A DAS28 greater than 5.1 indicates patients are in the active disease phase, less than 3.2 suggests low disease activity, and less than 2.6 suggests remission. More refined DAS instruments for subtypes of CA exist as well, e.g., the BASDAI (Bath Ankylosing Spondylitis Disease Activity Index) [20] features six items measuring discomfort, pain, and fatigue specifically for these patients. The BASFI (Bath Ankylosing Spondylitis Functional Index) [21] features 10 questions regarding physical activity.

2) Profiling on the basis of Avoidance-Persistence models: Based on the observation that some persons develop a "more substantial psychological overlay to their pain then others," [22] in 1983 already, the Fear-Avoidance model (FAM) was formulated [23]. In particular, FAM highlights how negative appraisal of pain may lead to catastrophic cognitions. This

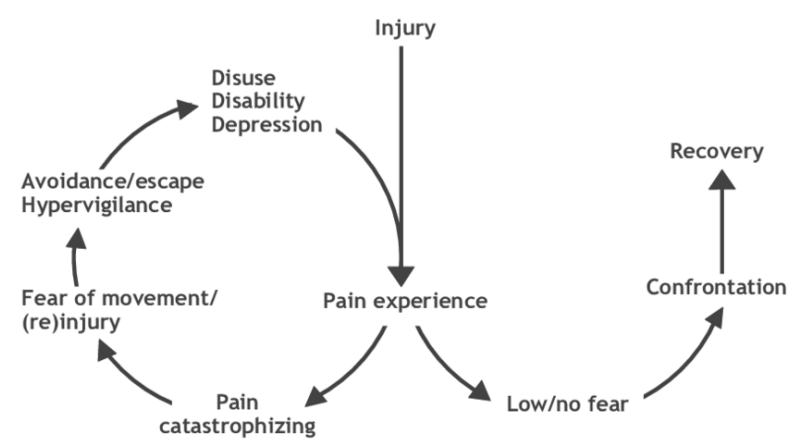

Fig. 1. The Fear-Avoidance model by [23], [28].

then leads to fear and avoidant behaviors, and possibly hypervigilance to bodily sensations, see Fig. 1. In contrast, when pain is perceived as non-threatening, persons are likely to confront this pain and maintain daily activities and movement, through which functional recovery is promoted.

While not developed for patients with CA per se, FAM has received much recognition [23], [24], and many health professionals in CA tailor interventions based on the level of avoidance versus confrontation of pain. Persons are reassured and encouraged to remain engaged in activity despite pain [25], to avoid catastrophizing and triggering this fear avoidance spiral.

On the basis of FAM, six distinct activity patterns among patients with chronic musculoskeletal pain can be distinguished: pain avoidance, activity avoidance, task-contingent persistence, excessive persistence, pain-contingent persistence, and pacing (dividing daily activities into smaller tasks) [26]. Based on the combination of different of these activity patterns, different patient types can be distinguished. Noteworthy is that recent models [24], [27], next to fear-avoidant types, also highlight the existence of a patient type that shows excessive persistence, not respecting physical limits. Excessive persistence is dysfunctional as well, as it may result in a number of potential injuries to muscle fibers, nerves, bones, and ligaments. For clinicians, it is important to understand the patient and to negotiate between reassuring and encouraging patients to confront pain, yet not to persist in excessive, detrimental activity.

In sum, different theories and models have been found helpful by therapists and physicians to tailor interventions for different patients with CA patients. Given the need for health professionals to understand the type of patient in front of them and to tailor intervention strategies towards the patient type, this is equally valid for interactive technologies designed to support patients with $\mathrm{CA}$ in their disease management.

\section{B. Interactive Technologies to Support and Motivate Patients with CA}

There have been several attempts at supporting physical therapy for patients with CA through technology, aiming to lower the effort needed to participate in physical therapy and 
to motivate patients to sustain in disease management. Many older studies relied on a personal computer to deliver physical therapy through a website, e.g., [29]-[32], via an additional pedometer to log steps [31], or using a webcam to providing feedback during physical therapy execution [33]. With the rise of mHealth, also smartphone apps for patients with CA have boomed. Several researchers have investigated what patients with CA want from such apps.

Revenäs et al. [34] collaborated with 26 patients with rheumatoid arthritis, on a mobile internet service, and identified several key features to support physical activity for patients with rheumatoid arthritis: up-to-date and evidencebased information, self-regulation tools, social interaction, personalized set-up, attractive design, and access to the internet service. Geuens et al. [35] also investigated patients with CA preferences for mHealth features to support self-management. They found that patients preferred features related to supporting active and direct disease management, (e.g., medication intake and detecting and alarming of bad posture), helping them to keep a close watch on their disease status and inform their health professionals (e.g., providing a means to log and report disease-related data) and receiving personalized information (e.g., offering tailored information based on the patient's health data). Gupta et al. [9] found that in order to help patients with CA in self-managing their physical activity behavior they especially preferred visualizations of their progress towards daily physical activity goals. These authors also articulated that personalization of treatment strategy is paramount, due to differences in disease related parameters and personal goals.

This finding is also echoed by Swann-Sternberg and colleagues [8], [36] who investigated how to build technologies to support people with chronic and musculoskeletal pain and particularly focused on removing the psychological barriers for movement. The authors also found personalization in terms of types of pain, abilities, goals, types of motivation, to permeate throughout all other findings [36].

Past research has also attempted to classify persuasive and motivational features in technology, (e.g., websites [37], mobile applications [37], [38], and wearables [39]) at a more generic level. Taxonomies such as the Behavior change taxonomy [40], Persuasive System Model [41], or the Motivational Design Lenses of mHealth [42] are models or frameworks that encompass a collective of strategies, heuristics or features that aim to motivate diverse users to exhibit or sustain a certain behavior. Such strategies may vary from providing task support [43], over social influence principles [44] to gamification [45]. While not discounting the value of such work, there may be a complementary need for a more situated understanding of motivational strategies, and how they are perceived by a diversity of users [46], [47], to account for the heterogeneity in audiences and contexts-of-use.

In order to address the need of health professionals to profile the patient with CA [26], [27] to devise an appropriate intervention strategy, and in order to answer to the call of health app

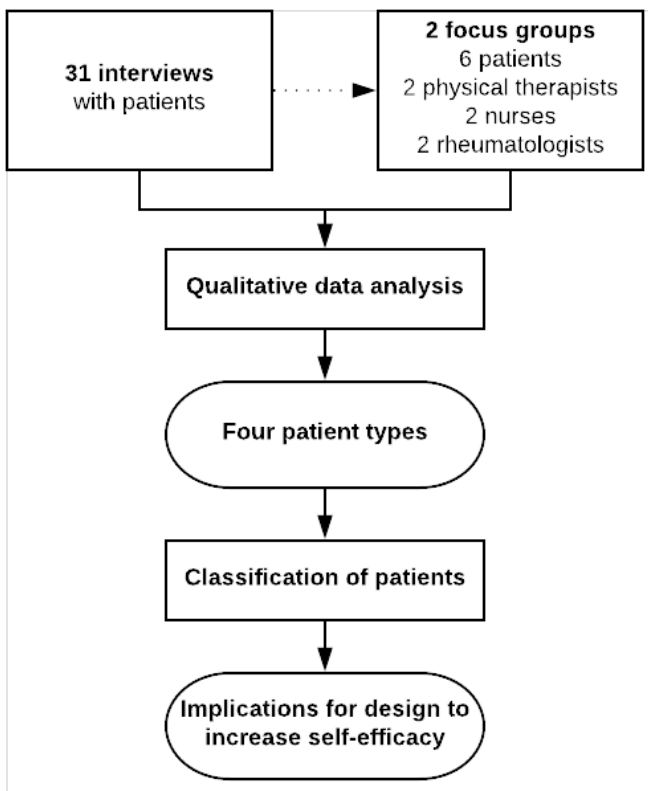

Fig. 2. Flowchart illustrating the different steps carried out during the research.

designers to personalize interactive technologies for patients [9], [35], [48], we set forward to investigate differences among patients with $\mathrm{CA}$ and their preferences for motivations features embedded in mHealth.

\section{METHOD}

The research described in this paper aims to study differences in patients with $\mathrm{CA}$ and preference of motivational mHealth features, by means of an open, explorative qualitative study with patients with CA and health professionals. As illustrated in Fig. 2, 31 interviews with patients with CA were conducted. This relatively high number of participants (for a qualitative study) was to ensure that the heterogeneity present among patients with CA was sufficiently captured, and thus that saturation in the analysis could be reached. In parallel, two focus groups with six patients with $\mathrm{CA}$ and six healthcare professionals (nurses, physical therapists and rheumatologists) were organized.

\section{A. Procedure Semi-Structured Interviews}

The interview started with open questions about how patients were managing their disease, with the aim of gaining insight in what patients did to manage their disease and why they carried out specific disease management actions. After the semi-structured start of the interview, the interview continued by asking to rate 26 possible motivational features of mHealth applications for patients with CA, on a Likert scale ( 1 being least favorable, 5 being most favorable). These features were selected from the Lenses of Motivational Design 
for mHealth [42], which lists 26 motivational principles in seven different categories. Each motivational principle was contextualized for patients with CA via a short description of a possible implementation, which was read aloud (see Table I). After patients were asked for a score, they were also asked why they were giving this score and were encouraged to provide additional information. This allowed investigating the underlying reasons affecting the preference for each feature. The duration of the interview varied between 8 and 31 minutes. The full details of the quantitative results are reported in [35].

Participants Interviews: Patients for the interviews were recruited at the outpatient CA clinic at the University Hospital Gasthuisberg in Leuven, Belgium. Ethical approval for this research was obtained from the Social and Societal Ethics Committee of KU Leuven with protocol number S-59012 Inclusion criteria were 1) participants should be at least 18 years old and 2) participants should be diagnosed with CA. Participants were given information about the study and were asked to sign a consent form detailing the collection, processing, and analysis of data. In total, thirty-one patients with CA (14 females), aged 23-71 ( $\mathrm{M}=51, \mathrm{SD}=12.16)$, were interviewed over the course of four months. All interviews were conducted one-on-one although during three of the interviews a translator or family member was present. Since participants were registered and in timely follow-up at the university hospital, we also had access to their disease status as reported by their rheumatologist (see Table II). BASDAI features six items measuring discomfort, fatigue, and pain on a 1-10 Likert scale, 10 is worst. BASFI features 10 items regarding physical activity on a $1-10$ scale, 10 is worst.

\section{B. Procedure of Focus Groups}

In addition to the individual interviews, two focus group sessions were organized. We followed the focus group protocol from [49] to mitigate unbalanced power dynamics which might occur in focus groups between healthcare professionals and their patients. In particular, six themes were introduced and attitudes were asked for: sensors, other data, sharing, short term feedback, long term feedback and therapy.

Participants for the focus group sessions were purposefully recruited from the pool of interview participants, as well as via referrals from a national patient organization for patients with CA. Medical personnel was recruited via purposeful sampling from the rheumatology division's staff at the University Hospital Gasthuisberg in Leuven, Belgium. In total, six patients with CA, two rheumatologists, two physical therapists and two specialized nurses participated in the focus groups.

\section{Analysis of the Interviews and Focus Groups}

All interviews and focus groups were recorded and transcribed verbatim. Afterwards, the qualitative data was coded according to the QUAGOL [50] method, bearing resemblance to grounded theory in that it is characterized by an iterative process of data analysis and interpretation.
TABLE I

STATEMENTS USED TO POLL FOR PATIENT PREFERENCES.

\begin{abstract}
ABILITY
Reduction: You are asked to enter measurement results in an app. The app automatically calculates several useful diseaserelated scores instead of you having to calculate these scores by hand.

Tunneling: You want to be able to walk a distance of five miles in a few months. The app calculates the right exercise schedule to guide you towards this goal.

Instruction: You are required to perform a set of exercises. The app provides detailed instructions on how to perform each exercise.

Goal Setting: You are able to choose your goal and the app will guide you towards this goal.

Rehearsal: Sensors measure whether you are executing an exercise the right way. You are able to perform the exercise a few times before the measurement is actually started.

Reminders: You are required to take your medication at fixed intervals. The app reminds you when you need to take your medication.
\end{abstract}

\section{INFORMATION}

General Information: You are able to read general information about arthritis in the app.

Macro Tailoring: The information in the application is specific for your type of arthritis.

Micro Tailoring: The information in the application is specific to you personally.

Simulation: The app is able to predict possible causes of pain from the collected data.

\section{AWARENESS \& INSIGHT}

Logging: You are able to save information about your condition in the app to show to your physician.

Tracking: The app automatically collects data relevant to your disease.

Self-monitoring: You are able to consult graphs and data based on your own data.

\section{INCENTIVIZATION}

Rewards: You are able to collect rewards based on your execution of exercises.

Praise: You are encouraged during your physical therapy through motivational messages.

Recognition: The app shows other users that you have been taken the most steps this week.

\section{SOCIAL SUPPORT}

Social identification: You are able to view limited data of other users with the same condition.

Social comparison: You are able to compare yourself to other users.

Competition: You are able to challenge other users to, for example, walk the longest distance.

Cooperation: You are able to work together with other users to achieve a common goal.

Social facilitation: While you are executing your exercises, family and friends are able to send you motivational messages.

\section{CREDIBILITY \& STYLING}

Personalization: You are able to personalize the app, for example, change colors, set a profile picture, choose what is shown.

Suggestion: The app detects bad posture and suggests to correct your posture.

Verifiability: The app shows scientific articles that describe the design and development of the app.

Expertise: The app shows physicians, therapists and researchers who helped create the app.

Surface credibility: The app does not contain advertisements. 
TABLE II

PARTICIPANTS IN INTERVIEWS AND FOCUS GROUPS. (*: ALSO PARTICIPATED IN FOCUS GROUP)

\begin{tabular}{|c|c|c|c|c|c|}
\hline Participant & Sex & $\begin{array}{l}\text { Age } \\
\text { (years) }\end{array}$ & $\begin{array}{l}\text { Diagnosis } \\
\text { (years) }\end{array}$ & BASDAI & BASFI \\
\hline Patient 1 & M & 48 & 33 & 2.60 & 2.50 \\
\hline Patient 2 & $\mathrm{~F}$ & 48 & 30 & 7.80 & 6.90 \\
\hline Patient 3 & $\mathrm{M}$ & 54 & 26 & 3.30 & 5.70 \\
\hline Patient 4 & $\mathrm{M}$ & 59 & 9 & 0.80 & 0.50 \\
\hline Patient 5 & $\mathrm{~F}$ & 34 & 9 & - & - \\
\hline Patient 6 & $\mathrm{M}$ & 55 & 39 & 1.80 & 5.70 \\
\hline Patient 7 & M & 66 & 47 & 3.40 & 4.10 \\
\hline Patient 8 & $\mathrm{~F}$ & 71 & 46 & 4.40 & 5.00 \\
\hline Patient 9 & $\mathrm{M}$ & 47 & 22 & 6.40 & 9.50 \\
\hline Patient 10 & $\mathrm{~F}$ & 51 & 18 & 3.60 & 2.90 \\
\hline Patient 11 & $\mathrm{M}$ & 45 & 10 & 3.50 & 5.20 \\
\hline Patient 12 & M & 23 & 10 & 0.80 & 0.00 \\
\hline Patient 13 & $\mathrm{~F}$ & 58 & 27 & 7.00 & 6.70 \\
\hline Patient 14 & $\mathrm{M}$ & 63 & 44 & 0.00 & 0.00 \\
\hline Patient $15^{*}$ & $\mathrm{~F}$ & 41 & 12 & 7.10 & 8.10 \\
\hline Patient 16 & $\mathrm{~F}$ & 59 & 14 & 6.50 & 7.30 \\
\hline Patient 17 & $\mathrm{~F}$ & 56 & 7 & 4.00 & 2.20 \\
\hline Patient 18 & $\mathrm{~F}$ & 69 & 34 & 2.70 & 1.90 \\
\hline Patient 19 & $\mathrm{M}$ & 38 & 1 & - & - \\
\hline Patient 20 & M & 39 & 15 & 1.10 & 1.40 \\
\hline Patient 21 & $\mathrm{~F}$ & 38 & 14 & 3.70 & 2.30 \\
\hline Patient 22 & $\mathrm{M}$ & 57 & 14 & 5.80 & 5.70 \\
\hline Patient 23 & $\mathrm{~F}$ & 41 & 16 & 5.40 & 4.40 \\
\hline Patient 24 & $\mathrm{~F}$ & 61 & 9 & 7.00 & 5.90 \\
\hline Patient 25 & $\mathrm{~F}$ & 46 & 14 & 5.10 & 5.30 \\
\hline Patient 26 & M & 29 & 8 & 1.30 & 0.00 \\
\hline Patient $27^{*}$ & $\mathrm{M}$ & 59 & 41 & 4.70 & 2.20 \\
\hline Patient 28 & M & 54 & 27 & 7.20 & 6.40 \\
\hline Patient 29 & M & 49 & 27 & 3.80 & 7.00 \\
\hline Patient 30 & $\mathrm{~F}$ & 67 & 14 & 6.90 & 6.90 \\
\hline Patient $31^{*}$ & M & 48 & 27 & 1.20 & 1.90 \\
\hline Patient $32 *$ & $\mathrm{M}$ & 69 & - & - & - \\
\hline Patient $33^{*}$ & $\mathrm{~F}$ & 45 & - & - & - \\
\hline Patient $34^{*}$ & M & 63 & - & - & - \\
\hline Nurse 1 & $\mathrm{~F}$ & - & - & - & - \\
\hline Nurse 2 & $\mathrm{~F}$ & - & - & - & - \\
\hline P.T. 1 & M & - & - & - & - \\
\hline P.T. 2 & $\mathrm{M}$ & - & - & - & - \\
\hline M.D. 1 & $\mathrm{~F}$ & - & - & - & - \\
\hline M.D. 2 & $\mathrm{M}$ & - & - & - & - \\
\hline
\end{tabular}

1) Establishing patient types: First, 15 interviews were coded twice in an open-coding round by one researcher to establish a coding dictionary. During this initial coding, codes from previously coded interviews were constantly compared to new interviews. After coding these 15 interviews, three interviews were independently coded by two other researchers, one researcher with expertise in grounded theory approaches and one physical therapist, experienced with qualitative research and psychotherapy. This was done to adjust and improve the coding tree. Next, the entire dataset was coded using the improved coding dictionary. In a next step, codes were organized into overarching concepts. These concepts were discussed and refined in two iterations with the other researchers. A next iteration was carried out, comprised of selective (i.e., axial coding) where the prior interviews were selectively coded, as well as the new data from both focus group sessions. Four types of patients were identified (see Fig. 3). Each interview was then again coded by two researchers independently, who analyzed all transcripts and classified all patients. Inter-coder reliability was assessed using Cohen's Kappa and was found to be K.904 which is excellent [51].

2) Establishing preferences for motivational design: After categorizing each patient with respect to patient types, adhering to the method of constant comparison [50], each interview was again coded using these categories and their underlying concepts. Collected data about how they rated each of the 28 motivational principles was re-analyzed. The average Likert rating of each motivational principle for a certain patient type was compared to the average rating of all patients of a motivational principle, allowing to establish the motivational principles that were favored or disliked by each patient type. From this re-inspection, the different preferences and implications for design were identified.

\section{RESULTS}

In this section, we first discuss the patient typology derived from the qualitative analysis. Next, we discuss the preferences and attitudes of these four CA types towards motivational mHealth features.

\section{A. CA Patient Typology}

As can be seen from Table II, patients with CA in this study differed significantly from each other with respect to their medical disease status. Scores varied from 0.00 to 7.20 on the BASDAI $(\mathrm{M}=4.02, \mathrm{SD}=2.26)$ and 0.00 to 9.50 on the BASFI $(\mathrm{M}=4.02, \mathrm{SD}=2.66)$. Disease duration was found to be between 3 and 49 years $(M=23, S D=13$ years). However, patients with CA differed not only in medical disease status but equally in self-reported pain severity and level of disability, their willingness to engage in activity, perception of control over the disease, and their mood. On the basis of these differences, we identified four patient types (see Fig. 3).

\section{Patients of the Defeatist type}

This first patient type is characterized by a low medical disease status, high levels of pain and high levels of disability. Patients of the Defeatist type are no longer willing to engage in activity and voice a low belief in arresting the disease and gaining back control over their life. Not surprisingly, these patients appear depressed and frustrated over their situation.

Patients of the Defeatist type have high BASDAI and BASFI scores (ranging from 6.40 to 7.00 on the BASDAI and 5.90 to 9.50 on the BASFI). They have had the disease for many years and have experienced it worsen over the years without any improvement. 


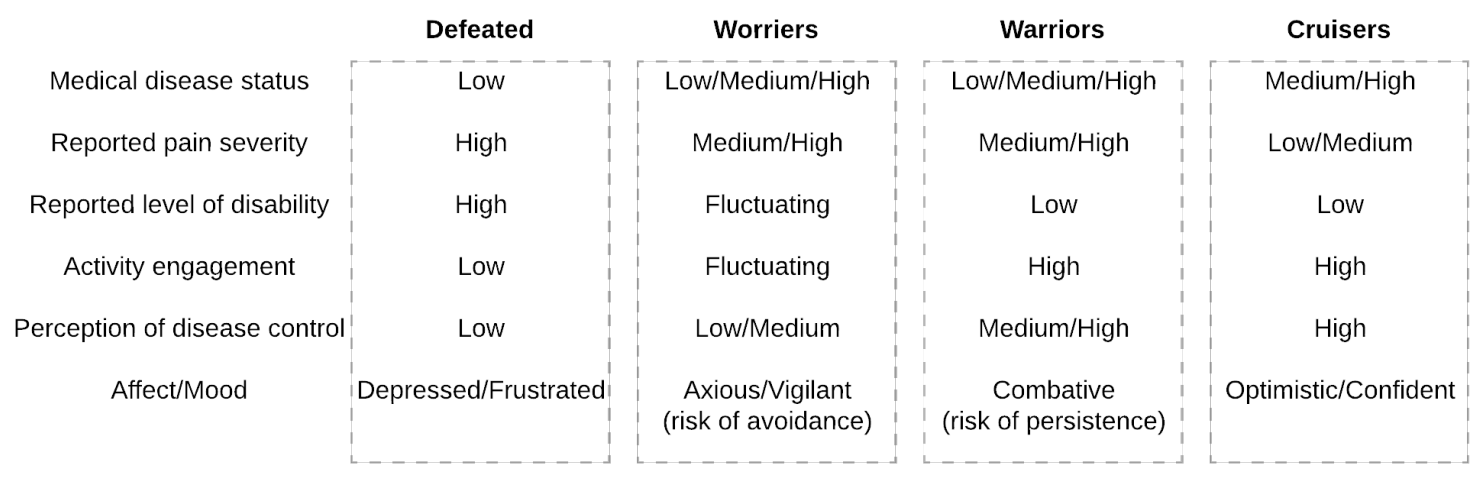

Fig. 3. Four types of patients with CA and the dimensions influencing them.

P9: "In the past, I had the feeling I would never walk again. I stayed like that. The rheumatologists have been looking for a solution for four, maybe five years... still nothing. I receive those injections but [my condition] still hasn't improved."

P19: "It's too late for physical therapy. My entire spine is fused together."

As a result, patients of the Defeatist type are characterized by a disbelief that disease management actions would help and express a reluctance to engage in self-management activities.

P9: "I got prescribed physical therapy but I didn't see any improvement and it only caused more pain, so I stopped going. It costs me and the society money and I don't see improvement, so I don't go."

Instead, they express a reliance on medication to lower pain and mitigate disease progress.

P9: "I take so many pills that I need to eat a sandwich just to be able to swallow all of them."

These patients have become fearful and/or 'avoidant' of pain, which has a significant impact and limits them in their daily physical activity and mobility.

P9: “Today, I'm feeling well, I'm not feeling any pain. But there is no way that I would force myself to do exercises today and then spend the next three months in my bed due to pain. When I'm feeling well, there's not a chance that I will do physical therapy."

P24: "When I move, I feel pain, so I rest most of the time."

In sum, patients of the Defeatist type have given up on improving their disease status via physical therapy exercises. In fact, they avoid activity for the fear of pain. They feel as nothing helps and mainly resort to medication to numb the pain they frequently experience.

\section{Patients of the Worrier type}

The second patient type is characterized by medium medical disease status, fluctuating moments of high pain severity and high disability. However, patients of the Worrier type are still willing to engage in activity at moments where the disease and pain is less acute. Patients express less control over how the disease will progress and report anxiety over interactions with medication and comorbidities with other diseases. Patients of the Worrier type also demonstrate vigilant behaviors.

Patients of the Worrier type have divergent BASDAI and BASFI scores (ranging from 0.80 to 3.40 on the BASDAI and 0.00 to 4.10 on the BASFI), but most importantly, they emphasize the fluctuations and tend to focus on the bad moments. The pain, and particularly the fear of pain has a significant impact on them and limits them in their willingness to engage in daily physical activity and movement.

P16: "When I feel pain, I lie on my couch and watch TV. I don't have the courage anymore. I do only the absolutely necessary like cooking and the laundry. I try to walk my dog as minimally as needed, very short and other people shouldn't bother me." P28: "Of course, you think about it a lot. It's there when you wake up and when you go to sleep. [...] About the pain, your entire body is fused together."

Patients also expressed the catastrophizing and the anxiety on how their disease will progress over time and how it will affect their life and the life of the people around them.

P3: “Also, when you feel something you haven't felt before. If I would be able to compare my symptoms to those of other patients... Now, I contribute all pain I feel to my condition but maybe the cause is something else that isn't related to my disease..." P24: "I think about it a lot. I'm afraid my condition will only get worse. My husband helps me a lot, but he's not getting younger. That's something that's always on my mind, how my life will continue."

Worriers also show a need to be reassured that they are taking appropriate action, both with respect to implications on the short term and long term. Patient 3, for example, describes the need to write down effects to be able to share this with 
their MD, and their wish to compare their symptoms to other patients:

P3: "I started writing downside effects since I started that new drug. Just for a few days. That way I can ask the doctor if other patients also experience these symptoms. I take painkillers every day? When you feel that it's not enough, you take more. But if I take more, my stomach starts acting up and I get even more problems."

During the focus groups, these patients insisted on a way to communicate with their physician.

P12: "I write down all my symptoms in a sort of diary. It helps me to manage my disease. Actually, I write down more in my diary than I mention [at the hospital]."

In sum, Worriers are motivated to manage disease status, but mostly through circumstantial actions such as vigilant monitoring and charting of disease parameters and seeking reassurance. They do engage in physical therapy but remain wary of the possible consequences.

\section{Patients of the Warrior type}

A third patient type is characterized by medium medical disease status, similar to patients of the Worrier type. They also report momentary high levels of pain, but they do not report high levels of disability. These patients show a high willingness to remain engaged in activity, despite the presence of pain. They are confident, perhaps overconfident, to exercise, and show an overall combative mood.

Patients of the Warrior type have divergent BASDAI and BASFI scores (ranging from 1.80 to 7.10 on the BASDAI and 1.40 to 8.10 on the BASFI). They also report high levels of pain severity, but do not find themselves limited in their physical activities. In fact, they actively seek out physical movement as they are convinced of the beneficial effect.

P15: "I try to walk as much as possible. They are mostly short walks. I bought a small dog especially for that purpose, so I would be obliged to get out the door and go walking."

Patients of the Warrior type endure and confront pain.

P11: "I am not prescribed physical therapy but I do it anyway. I try to do that three times a week because it reduces pain and helps me bear the pain. I'll also experience pain less frequently. The stiffness afterward is no problem. I'm also motivated to keep going because I really see improvement. I also try not to lose courage. I try to do everything I did before."

These patients with CA follow their prescribed therapy and express good motivation and confidence in knowing what to do to improve their disease status. Patient 23 describes:

P23: "Some weeks are difficult when you experience a lot of pain. Pain is tough, especially at night. Stress is also an important factor for me. [...] Physical therapy is essential for me. I attend physical therapy three times a week and over the years I have put together a whole catalog of physical therapy exercises that I can do at home each day. I also do yoga each week, that's something I would recommend to other patients as well."

However, excess physical activity can also be detrimental to disease status and can lead to increased pain. Patients of the Warrior type should be able to identify excess physical activity or should be reminded during physical activity not to exaggerate. During the focus group one of the health professionals articulated this to one of the patients:

P.T.1: "Compare [your body] to a car that needs fuel.

You should have a warning light that tells you when you need to refuel."

In sum, patients of the Warrior type also acknowledge the effect of pain on their life, but rather than engaging in pain avoidance and/or vigilant behaviors, they engage in physical actions that can improve their disease status. However, Warriors show a tendency to ignore pain warnings, and may engage in excessive physical activity.

\section{Patients of the Cruiser type}

The last patient type is characterized by medium or low medical disease status, low levels of pain severity and disability. Cruisers show a high willingness to engage in activity, and perceive themselves as being in control, having a confident, optimistic attitude towards the disease.

Patients of the Cruiser type have BASDAI and BASFI scores ranging from 0.80 to 4.40 on the BASDAI and 0.50 to 7.00 on the BASFI. These patients are rarely bothered by their condition and seem like they cruise through life, often unmindful of their condition.

P18: "I don't think about my condition that often.

I'm lucky that my medication works."

P21: "It has become a recurring theme in my life, but it has become a routine. I can do everything I want; I only experience a bit of pain from time to time."

Patients of the Cruiser type do express moments of pain but do not show worry.

P22: "I only think about my condition when I feel pain. I don't worry about it. I don't think about my condition, absolutely not. It's not like that is going to solve anything. I have been living with my condition since '92, you don't think about it, it has become a routine. I don't complain when my knees are swollen, I visit the doctor and take some more medication."

These patients with CA seem to have learned to manage their disease and cater their life around the limitations that the disease provides them. While Cruisers express less motivation to intentionally act in disease management activities such as physical therapy, it should be noted that they often have developed beneficial habits that include physical movement. 
P21: "I have to participate in physical therapy but that's mainly to reduce stiffness. So I don't really do any physical therapy without my therapist. Apart from yoga. And some swimming and cycling, typically an hour each week."

They have adapted life and or medication regimen in such a manner in such a manner that they do not put much (mental) energy in the disease. Patient 32 describes this knowledge when asked about pain experienced after strenuous physical activity:

P32: "I will never reach that point of experiencing pain because I have learned what my limitations are."

In sum, patients of the Cruiser type have developed a beneficial lifestyle that reconciles the disease with activities they find meaningful. Different from Warriors, they seek to minimize their effort in disease management.

\section{B. Preferences for Motivational Features According to the Different Patient Types}

From the above analysis, it becomes apparent that motivational mHealth features may need to be tailored towards the different patient types. Due to differences in medical disease status but equally differences in pain severity and level of disability, willingness to engage in activity, perception of control over the disease, and mood, patients will respond differently to the different motivational features. Here we discuss how patients with CA rated the motivational features differently between patient types, organized according to the categories of the Lenses for Motivational Design for mHealth in Table I.

\section{Ability}

This category groups motivational features that support patients with CA active behaviors to engage in physical activities (see Table I).

In general, except for the Defeatist, patient types were positive toward features that support physical activity. Patients from the Defeatist type emphasized that they would not engage in physical activities.

I: "You want to be able to walk a distance of five miles in a few months. The app calculates the right exercise schedule to guide you towards this goal." P9 (Defeatist): "For my personally? A one, for sure I am not going to do it."

P19 (Defeatist): (ironically) "This could be interesting. A five? Not for me! But for other people perhaps? [...] I will explain it this way: today I am good, I am not in pain, so I am not going to force myself today to do exercises in which I can lie down on a bed for the next three months. If I'm feeling good, there's not one hair on my head thinking of doing exercises. Up, down... All I know. Is that I am so grateful that I have not suffered from my back for two months now."
Patients from the Worrier type were more positive towards ability related motivational features, yet they expressed doubt about the ingenuity of such technological system and voiced a preference for support by physical therapists.

\section{I: "Sensors measure whether you are executing an} exercise the right way [...]"

P3 (Worrier): "I don't know if that... then it must be very ingenious, [...] I have some reservations about it because the physical therapist always says, 'You can do that [Exercise], but if you feel that you can't go any further, then you stop.' But if that app says, further, further... I would personally think that that is difficult to make. There are so many variants of arthritis, it may be that it is difficult to build."

P5 (Worrier): "I am more a person of personal contact, an app... that is not really that..."

Patients of the Warrior type and Worrier type were most in favor of such strategies.

P23 (Warrior): "At our workplace, they tried that for a while, something on their back that detected their posture and indicated whether it was good or bad. I think that would be a good one for me to have. So in that sense these are things that I would say yes to."

P25 (Cruiser): "These exercises, the posture, I would for sure pay attention... If I am sitting wrongly (imitates computer voice): 'Sit up straight!'”

\section{Disease insight}

This category groups features that allow patients with CA to keep a closer watch on their disease and communicate to professionals (see Table I). These features were particularly liked by Worriers. They liked being able to document their disease progress and being able to use this in communication with physical therapist, to further their understanding.

P3 (Worrier): "For sure, this seems handy. Because now, every two weeks I take some notes, because of side-effects, but this way, you can constantly save information."

P12 (Worrier): "This seems useful because we only see the doctor every couple of months. This way, I can better indicate how I feel in the long run. Then, they [the health professionals] know more and can make my treatment more aligned."

It appears that such strategies may help reassure and avoid catastrophizing. However, such strategies may equally feed into hyper-vigilance. Strategies to further disease insight were also particularly liked by Warriors. Defeatist and Cruisers were somewhat less enthusiast about this category, but for very different reasons. Whereas Defeatist indicated they saw no use for this out of general frustration, Cruisers saw no use as they were not bothered much by their disease.

I: "The app automatically calculates several useful disease-related scores instead of you having to calculate these scores by hand." 
P9 (Defeatist): "Sure, go ahead (ironically) I don't care at all. Because I am high on Morphine. For me, not, I will not do this anyway."

P32 (Cruiser): "No, and why won't I do this?... Because it doesn't bother me. I have a high blood pressure and the doctor said, measure your blood pressure regularly. That I will do. But for my back? It doesn't bother me. So then, I will not make an effort to $\log$ all this."

\section{Information}

This category groups those features that provide either general, tailored (disease-related, at the group level), or personal information on arthritis to the patient. Here scores vary depending on the degree of how tailored information is offered. Although patients of the Defeatist and Worrier type gave high scores to motivational features in the information category, there were some doubts as to whether the provided information would be accurate.

P9 (Defeatist): “I don't believe that [that the app can tailoring information to an individual level]. There is not a single person in this hospital who can tell me what is causing my pain so I don't think an app would ever be able to provide me with that type of information."

P3 (Worrier): "I would just Google information. [About more tailored information] I think physicians have to evolve to be able to provide me with personalized information. They always tell me they 'suspect this and that' but they are never sure. So if a physician isn't able to be accurate, I don't think an app could be."

Some patients with CA relied on their rheumatologist to provide them with information and only trusted that information.

P5 (Worrier): "I'd rather ask this kind of information from my physician. Other than that, I don't really need information."

Contrary to P5, P3 (see above) and P23 do not rely on their physician for information.

P23 (Warrior): "[About general information] I can just get that information somewhere else."

Patients of the Cruiser type were in favor of motivational features of the information category claiming the information to be useful for them.

P30 (Cruiser): "General information, I would give it 4 out of 5. Tailored for my kind of condition, I would give it 5 out of 5 . And personalized for me, even better also 5 out of 5 ."

\section{Incentivization}

This category groups features that aim to increase motivation by providing rewards or praise. All patient types except the Defeatist type were unanimous in their dislike for incentivization, claiming that they do not need to be motivated to adhere to therapy.
P18 (Cruiser): "Rewards are not necessary for me, I do my physical therapy because it's good for my health."

P3 (Worrier): "Those rewards are not going to take away my pain."

P30 (Warrior): "That's for children. Although, it might be useful for some people... maybe children with CA."

\section{Social support}

This category contains features related to interaction, sharing, cooperating and comparing with others through an mHealth application. These features scored lowest of all categories. Patients of the Worrier, Warrior and Cruiser types want to keep their condition private and don't need others to support them in their therapy.

P22 (Warrior): "When I'm exercising, I'm exercising for myself. I don't need to involve others."

P3 (Worrier): "The people in my surroundings know my condition [...]. I don't want to bore them with my condition."

P26 (Cruiser): "This is a medical condition and is differently for everyone. I would not like to share it on the internet."

P9 (Defeatist): "That's something I would love to see! I have been in the hospital for nine months and not even my own mother visited me. So I don't think anyone would want to interact socially."

\section{Credibility \& Styling}

This category contains features related to how the application can increase perceived credibility and or be styled to personal liking. Scores diverged for the different features: whereas surface credibility was highly valued by all patients, for other features patients were indifferent. Among patient types, few differences were found.

\section{I: You are able to personalize the app, for example change colors [...]}

P18 (Cruiser): "This is OK for me, but it is not necessary, a 3."

I: The app shows physicians, therapists and researchers who helped create the app.

P3 (Worrier): "I only know one rheumatologist in one hospital. If this application would be used nationwide, I wouldn't recognize the people in the app anyhow."

\section{DISCUSSION}

Our findings show that a tailored approach is needed to target different types of patients with CA. These findings are in line with the conclusions of Gupta et al. [9], [52], Singh et al. [8], [48] and Swann-Sternberg et al. [36] all of whom, too, remark the importance of tailoring or personalizing treatment to individual patients or patient groups. Our typology also echoes the different profiling types that health professionals 
and in particular more recent models on Fear-Avoidance [22] and Endurance [24], [26], [27]. In addition to fear-avoidant types characterized by pain avoidance, activity avoidance, catastrophic cognitions and hyper-vigilance in lesser or milder degree (i.e., Defeatist and Worrier), we also found a patient type that risks excessive persistence (i.e., Warrior) and a patient type that has reconciled lifestyle with the disease through task-contingent persistence and pacing (i.e., Cruiser). Hence, the Defeatist-Worrier-Warrior-Cruiser typology confirms that a personalized, tailored approach is needed for mHealth interventions with patients with CA.

In addition, we have shown how these four types of patients exhibit different preferences for different motivational features. In the next section, we will address implications for design stemming from our findings. These recommendations provide persuasive strategies which target the needs of each patient type and aim to improve the patient's condition.

\section{A. Implications for Design}

Based on our typology of patients with CA, the following design implications can be derived.

1) Defeatist: Patients of the Defeatist type suffer from their disease and lack an overall sense of control. They feel as though their condition cannot improve. These patients might benefit from motivational features that increase their perceived control over the disease, e.g., providing small steps towards improvement or providing accurate and rapid feedback. Next, providing rewards and praise may stimulate them to make the first steps towards improvement. In addition, it may help reduce social isolation, i.e., by providing an online platform to talk and exchange information with peers.

2) Worrier: Patients of the Worrier type also suffer from low control over disease progress and particularly fear their disease will get worse. They often avoid exercising and physical activity for fear of increased discomfort from pain and stiffness. These patients may benefit from motivational features that offer reassuring information regarding disease progression and the beneficial effect of exercising on their disease status. In addition, these patients welcome disease status tracking features and features to log disease status, e.g., diaries, to be able to share this with physicians, to alleviate worries about their condition getting worse. However, caution is needed not to play into catastrophizing thoughts and to trigger hyper-vigilance. Finally, it is suggested to provide continuous guidance and feedback during exercises to reassure patients of the worrier type they are doing well, and to remind them of the positive effects of physical movement, both in the short run as well as long-term disease progression.

3) Warrior: Patients of the Warrior type try to do anything they can to improve their disease status. They are motivated to work hard to achieve this goal. These patients might benefit from motivational features that provide guidance and instruction during exercises to ensure that are conducting exercises correctly. Next, these patients welcome goal-setting as a means to extend physical exercise schemes, and to suit their ambitious therapy schedule. In addition, they like increased tracking and self-monitoring of their disease status, including simulations, to see what is beneficial. For this type, however, it is important to highlight excess activity, and to remind that this is detrimental to their disease status.

4) Cruiser: Patients of the Cruiser type do not think or worry about their disease often. They do not want to put much mental energy into their condition. These patients might benefit from motivational features that provide easy ways to track, for example, their infrequent pain episodes or to track appointments with their physician and physical therapist. To further reduce the effort needed to manage their disease, they welcome any automation in filling out questionnaires prior to consultation based on prior collected data. In addition, it is advised to allow them to disable features they feel do not benefit them (but might benefit other patient types, such as notifications about bad posture, sharing with others, and medication reminders).

\section{B. Limitations and Future Work}

While the patient typology presented here is tailored to patients with $\mathrm{CA}$, we argue that it may have broader use for patients characterized by chronic musculoskeletal pain. Hence, it may be useful for other chronic conditions that also induce (muscle) pain and require physical therapy. Such pathologies may equally benefit from targeting similar patient types, and thus, offers a way to tailor motivational strategies to deliver tailored mHealth interventions.

Future work could also investigate how such patient profiling relates to user-centered design approaches [53], and in particular the practice of creating personas, to inspire the design of interactive systems. At the same time, we acknowledge the limitations of this first exploratory, qualitative study. Further work is needed to validate the typology with different samples of (CA) patients. Moreover, we acknowledge that in this study, we focused on preferences of patients. Further research with health professionals is needed to understand how the tailoring of motivational features may interact with specific therapies. For example, the preference of Worriers for a diary and logging, may trigger hyper-vigilance and may, from a therapeutic perspective, be less desirable.

\section{CONCLUSION}

In this paper, we applied a qualitative study to establish four CA patient types. Moreover, we provide insight into which mHealth features are suitable for each patient type to support them in their disease management. We translated these insights into implications for the design of mHealth applications. We hope these findings may inspire healthcare informatics researchers to create apps tailored to the needs of patients with musculoskeletal diseases and chronic pain.

\section{ACKNOWLEDGMENT}

This work is part of the research project SPARKLE which is funded by the KU Leuven, Belgium. 


\section{REFERENCES}

[1] National statistics | data and statistics | arthritis | CDC. http://www.webcitation.org/73FudqgDN, 2018. Accessed: 2020-02-24.

[2] American Heritage Dictionary. The American Heritage Medical Dictionary. Houghton Mifflin Company, 2007.

[3] R. Campbell, M. Evans, M. Tucker, B. Quilty, P. Dieppe, and J. L. Donovan. Why don't patients do their exercises? understanding noncompliance with physiotherapy in patients with osteoarthritis of the knee. Journal of epidemiology and community health, 55(2):132-138, 2001.

[4] F. Viller, F. Guillemin, S. Briançon, T. Moum, T. Suurmeijer, and W. van den Heuvel. Compliance to drug treatment of patients with rheumatoid arthritis: a 3 year longitudinal study. The Journal of Rheumatology, 26(10):2114-2122, 1999.

[5] A. G. Schneiders, M. Zusman, and K. P. Singer. Exercise therapy compliance in acute low back pain patients. Manual Therapy, 3(3):147$152,1998$.

[6] WHO Global Observatory for eHealth and World Health Organization. MHealth: new horizons for health through mobile technologies. World Health Organization, 2011. OCLC: 759446797.

[7] Jonas Geuens, Thijs Willem Swinnen, Rene Westhovens, Kurt de Vlam, Luc Geurts, and Vero Vanden Abeele. A review of persuasive principles in mobile apps for chronic arthritis patients: Opportunities for improvement. JMIR mHealth and uHealth, 4(4):e118, 2016-10-13.

[8] Aneesha Singh, Annina Klapper, Jinni Jia, Antonio Fidalgo, Ana Tajadura-Jiménez, Natalie Kanakam, Nadia Bianchi-Berthouze, and Amanda Williams. Motivating people with chronic pain to do physical activity: Opportunities for technology design. In Proceedings of the 32Nd Annual ACM Conference on Human Factors in Computing Systems, CHI '14, pages 2803-2812. ACM, 2014.

[9] Ankit Gupta, Tim Heng, Chris Shaw, Linda Li, and Lynne Feehan. Designing pervasive technology for physical activity self-management in arthritis patients. In Proceedings of the 12th EAI International Conference on Pervasive Computing Technologies for Healthcare, PervasiveHealth '18, pages 1-10. ACM, 2018. event-place: New York, NY, USA.

[10] Sarah L. Gorst, Christopher J. Armitage, Simon Brownsell, and Mark S. Hawley. Home telehealth uptake and continued use among heart failure and chronic obstructive pulmonary disease patients: a systematic review. Annals of Behavioral Medicine: A Publication of the Society of Behavioral Medicine, 48(3):323-336, 2014-12.

[11] Gunther Eysenbach. The law of attrition. Journal of Medical Internet Research, 7(1):e11, 2005-03-31.

[12] Rita Orji, Julita Vassileva, and Regan L. Mandryk. Modeling the efficacy of persuasive strategies for different gamer types in serious games for health. User Modeling and User-Adapted Interaction, 24(5):453-498, 2014-12-01.

[13] Rita Orji, Gustavo F. Tondello, and Lennart E. Nacke. Personalizing persuasive strategies in gameful systems to gamification user types. In Proceedings of the 2018 CHI Conference on Human Factors in Computing Systems, CHI '18, pages 435:1-435:14. ACM, 2018. eventplace: Montreal QC, Canada.

[14] Robin De Croon, Alemitu Mequanint Bezabih, Jonas Geuens, Davina Wildemeersch, Dries Oeyen, Katrien Verbert, and Vero Vanden Abeele. Motivational design techniques to increase adherence to a telemonitoring therapy a study with adolescent pectus patients. In 2019 IEEE International Conference on Healthcare Informatics (ICHI). IEEE, 2019-06-13.

[15] Maurits Kaptein, Boris De Ruyter, Panos Markopoulos, and Emile Aarts. Adaptive persuasive systems: A study of tailored persuasive text messages to reduce snacking. ACM Trans. Interact. Intell. Syst., 2(2):10:1-10:25, 2012-06.

[16] Alan J Silman and Jacqueline E Pearson. Epidemiology and genetics of rheumatoid arthritis. Arthritis research \& therapy, 4(S3):S265, 2002.

[17] John J. Bonica. Bonica's Management of Pain. Lippincott Williams \& Wilkins, 2001. Google-Books-ID: TyNEicOiJqQC.

[18] Jessica C. Payne-Murphy and Abbie O. Beacham. Revisiting chronic pain patient profiling: An acceptance-based approach in an online sample. Clinical Psychology \& Psychotherapy, 22(3):240-248, 2015 06.

[19] Arthritis self management - arthritis self care. (Accessed on 05/02/2019)

[20] Sarah Garrett, Tim Jenkinson, L Gail Kennedy, H Whitelock, P Gaisford, and A Calin. A new approach to defining disease status in ankylosing spondylitis: the bath ankylosing spondylitis disease activity index. The Journal of rheumatology, 21(12):2286-2291, 1994.

[21] Andrei Calin, Sarah Garrett, Helen Whitelock, LG Kennedy, J O'hea, $\mathrm{P}$ Mallorie, and T Jenkinson. A new approach to defining functional ability in ankylosing spondylitis: the development of the bath ankylosing spondylitis functional index. The Journal of rheumatology, 21(12):22812285, 1994.

[22] J. Lethem, P. D. Slade, J. D. G. Troup, and G. Bentley. Outline of a fear-avoidance model of exaggerated pain perception-i. Behaviour Research and Therapy, 21(4):401-408, 1983-01-01.

[23] J. W. Vlaeyen and S. J. Linton. Fear-avoidance and its consequences in chronic musculoskeletal pain: a state of the art. Pain, 85(3):317-332, 2000-04.

[24] Monika I. Hasenbring and Jeanine A. Verbunt. Fear-avoidance and endurance-related responses to pain: new models of behavior and their consequences for clinical practice. The Clinical Journal of Pain, 26(9):747-753, 2010-12.

[25] Lance M. McCracken, Kevin E. Vowles, and Christopher Eccleston. Acceptance of chronic pain: component analysis and a revised assessment method. Pain, 107(1):159-166, 2004-01.

[26] Kevin E. Vowles, Lance M. McCracken, Charlotte McLeod, and Christopher Eccleston. The chronic pain acceptance questionnaire: confirmatory factor analysis and identification of patient subgroups. Pain, 140(2):284291, 2008-11-30.

[27] Monika I. Hasenbring, Dirk Hallner, and Adina C. Rusu. Fearavoidance- and endurance-related responses to pain: Development and validation of the avoidance-endurance questionnaire (AEQ). European Journal of Pain, 13(6):620-628, 2009.

[28] Maaike Leeuw, Mariëlle E. J. B. Goossens, Steven J. Linton, Geert Crombez, Katja Boersma, and Johan W. S. Vlaeyen. The fear-avoidance model of musculoskeletal pain: Current state of scientific evidence. Journal of Behavioral Medicine, 30(1):77-94, 2007-01-31.

[29] M. H. van den Berg, H. K. Ronday, A. J. Peeters, E. M. Voogtvan der Harst, M. Munneke, F. C. Breedveld, and T. P. M. Vliet Vlieland. Engagement and satisfaction with an internet-based physical activity intervention in patients with rheumatoid arthritis. Rheumatology, 46(3):545-552, 2007-03-01.

[30] Daniël Bossen, Cindy Veenhof, Karin EC Van Beek, Peter MM Spreeuwenberg, Joost Dekker, and Dinny H De Bakker. Effectiveness of a web-based physical activity intervention in patients with knee and/or hip osteoarthritis: Randomized controlled trial. Journal of Medical Internet Research, 15(11), 2013-11-22.

[31] Sarah L Krein, Reema Kadri, Maria Hughes, Eve A Kerr, John D Piette, Rob Holleman, Hyungjin Myra Kim, and Caroline R Richardson. Pedometer-based internet-mediated intervention for adults with chronic low back pain: Randomized controlled trial. Journal of Medical Internet Research, 15(8):e181, 2013-08-19.

[32] David A. Williams, David Kuper, Michelle Segar, Niveditha Mohan, Manish Sheth, and Daniel J. Clauw. Internet-enhanced management of fibromyalgia: a randomized controlled trial. Pain, 151(3):694-702, 2010-12.

[33] Michel Tousignant, Patrick Boissy, Hélène Moffet, Hélène Corriveau, François Cabana, François Marquis, and Jessica Simard. Patients' satisfaction of healthcare services and perception with in-home telerehabilitation and physiotherapists' satisfaction toward technology for post-knee arthroplasty: an embedded study in a randomized trial. Telemedicine Journal and E-Health: The Official Journal of the American Telemedicine Association, 17(5):376-382, 2011-06.

[34] Åsa Revenäs, Cathrin Martin, Christina H. Opava, Maria Brusewitz, Christina Keller, and Pernilla Åsenlöf. A mobile internet service for self-management of physical activity in people with rheumatoid arthritis: Challenges in advancing the co-design process during the requirements specification phase. JMIR Research Protocols, 4(3):e111, 2015-09-17.

[35] Jonas Geuens, Luc Geurts, Thijs W. Swinnen, Rene Westhovens, and Vero Vanden Abeele. Mobile health features supporting selfmanagement behavior in patients with chronic arthritis: Mixed-methods approach on patient preferences. JMIR mHealth and uHealth, 7(3):e12535, 2019.

[36] Tali Swann-Sternberg, Aneesha Singh, Nadia Bianchi-Berthouze, and Amanda Williams. User needs for technology supporting physical activity in chronic pain. In CHI' 12 Extended Abstracts on Human Factors in Computing Systems, CHI EA '12, pages 2441-2446. ACM, 2012. 
[37] Saskia M Kelders, Robin N Kok, Hans C Ossebaard, and Julia EWC Van Gemert-Pijnen. Persuasive system design does matter: a systematic review of adherence to web-based interventions. Journal of Medical Internet Research, 14(6):e152, 2012-11-14.

[38] Sitwat Langrial, Tuomas Lehto, Harri Oinas-Kukkonen, Marja Harjumaa, and Pasi Karppinen. Native mobile applications for personal well-being: A persuasive systems design evaluation. PACIS 2012 Proceedings, 2012-07-15.

[39] Thomas Fritz, Elaine M. Huang, Gail C. Murphy, and Thomas Zimmermann. Persuasive technology in the real world: A study of longterm use of activity sensing devices for fitness. In Proceedings of the SIGCHI Conference on Human Factors in Computing Systems, CHI '14, page 487-496, New York, NY, USA, 2014. Association for Computing Machinery.

[40] Susan Michie, Michelle Richardson, Marie Johnston, Charles Abraham, Jill Francis, Wendy Hardeman, Martin P. Eccles, James Cane, and Caroline E. Wood. The behavior change technique taxonomy (v1) of 93 hierarchically clustered techniques: Building an international consensus for the reporting of behavior change interventions. Annals of Behavioral Medicine, 46(1):81-95, 2013-08.

[41] Harri Oinas-Kukkonen and Marja Harjumaa. Towards deeper understanding of persuasion in software and information systems. In First international conference on advances in computer-human interaction, pages 200-205. IEEE, 2008.

[42] Jonas Geuens, Luc Geurts, Kathrin Gerling, Robin De Croon, and Vero Vanden Abeele. A dyad of lenses for the motivational design of mHealth: Bridging the gap between health theory and app design. In 2019 IEEE International Conference on Healthcare Informatics (ICHI). IEEE, 2019-06-12.

[43] Harri Oinas-Kukkonen and Marja Harjumaa. Persuasive systems design: Key issues, process model, and system features. Communications of the Association for Information Systems, 24(1):28, 2009.

[44] Robert B. Cialdini and Melanie R. Trost. Social influence: Social norms, conformity and compliance. In D. T. Gilbert, S. T. Fiske, and G. Lindzey, editors, The handbook of social psychology, Vols. 1 and 2 (4th ed.), pages 151-192. McGraw-Hill, 1998.

[45] Robin De Croon, Davina Wildemeersch, Joris Wille, Katrien Verbert, and Vero Vanden Abeele. Gamification and serious games in a healthcare informatics context. In 2018 IEEE International Conference on Healthcare Informatics (ICHI), pages 53-63. IEEE, 2018-06.

[46] Rita Orji, Regan L. Mandryk, Julita Vassileva, and Kathrin M. Gerling. Tailoring persuasive health games to gamer type. In Proceedings of the SIGCHI Conference on Human Factors in Computing Systems, pages 2467-2476. ACM, 2013.

[47] Maurits Kaptein, Panos Markopoulos, Boris de Ruyter, and Emile Aarts. Personalizing persuasive technologies: Explicit and implicit personalization using persuasion profiles. International Journal of Human-Computer Studies, 77:38-51, 2015-05.

[48] Aneesha Singh, Nadia Bianchi-Berthouze, and Amanda CdeC Williams. Supporting everyday function in chronic pain using wearable technology. In Proceedings of the 2017 CHI Conference on Human Factors in Computing Systems - CHI'17, pages 3903-3915. ACM Press, 2017.

[49] Jonas Geuens, Luc Geurts, Thijs W Swinnen, René Westhovens, Maarten Van Mechelen, and Vero Vanden Abeele. Turning tables: A structured focus group method to remediate unequal power during participatory design in health care. In Proceedings of the 15th Participatory Design Conference: Short Papers, Situated Actions, Workshops and Tutorial Volume 2, PDC '18, pages 4:1-4:5. ACM, 2018.

[50] Bernadette Dierckx de Casterlé, Chris Gastmans, Els Bryon, and Yvonne Denier. QUAGOL: A guide for qualitative data analysis. International Journal of Nursing Studies, 49(3):360-371, 2012-03-01.

[51] Matthew Lombard, Jennifer Snyder-Duch, and Cheryl Campanella Bracken. Content analysis in mass communication: Assessment and reporting of intercoder reliability. Human Communication Research, 28(4):587-604, 2002-10-01.

[52] Ankit Gupta, Tim Heng, Chris Shaw, Linda Li, and Lynne Feehan. Towards developing an e-coach to support arthritis patients in maintaining a physically active lifestyle. In Proceedings of the 12th EAI International Conference on Pervasive Computing Technologies for Healthcare, PervasiveHealth '18, pages 392-395. ACM, 2018. eventplace: New York, NY, USA.

[53] Alan Cooper, Robert Reimann, David Cronin, and Christopher Noessel. About face: the essentials of interaction design. John Wiley \& Sons, 2014. 